\title{
The New HZB X-Ray Microscopy Beamline U41-PGM1-XM at BESSY II.
}

$\underline{\text { Peter Guttmann }}^{1, *}$, Stephan Werner ${ }^{1}$, Frank Siewert ${ }^{1}$, Andrey Sokolov ${ }^{1}$, Jan-Simon Schmidt ${ }^{1}$, Matthias Mast $^{1}$, Maria Brzhezinskaya ${ }^{1}$, Christian Jung ${ }^{1}$, Rolf Follath ${ }^{2}$ and Gerd Schneider ${ }^{1}$

1. Helmholtz Zentrum Berlin für Materialien und Energie, BESSY II, Berlin, Germany.

2. Paul Scherrer Institute, Beamline Optics Group, Villigen, Switzerland.

* Corresponding author, peter.guttmann@helmholtz-berlin.de

At the BESSY II electron storage ring running by the Helmholtz-Zentrum Berlin (HZB) a transmission soft X-ray microscope (TXM) is operated very successfully for both tomographic imaging of cells $[1,2]$ and for NEXAFS studies in materials science [3-5]. Here, we present the setup of a newly designed beamline (Fig. 1) that will significantly enhance the performance of the HZB TXM. Faster data acquisition over the whole accessible photon energy range together with a possible extension into the tender X-ray range is given. Furthermore, within the photon energy tuning range, two new important absorption edges, namely sulfur and phosphorus, will become accessible for element-specific 3D imaging, in addition to those already available, e.g. at carbon, calcium, titanium and oxygen. Another advantage of this new beamline is that phase contrast X-ray microscopy for thicker specimens will become possible in the tender $\mathrm{X}$-ray photon energy range.

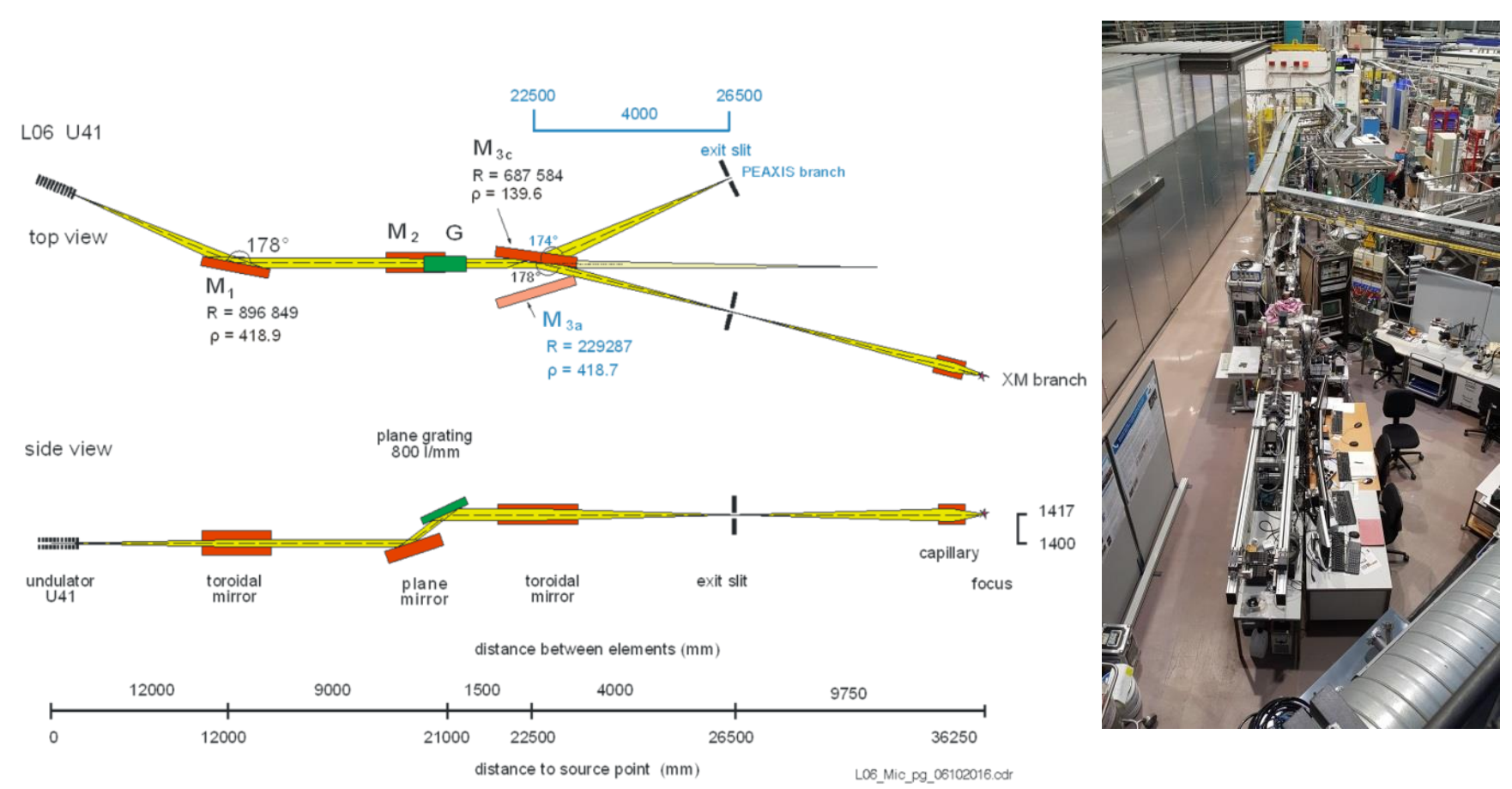

Figure. 1. Optical layout of the new HZB X-ray microscopy beamline U41-PGM1-XM (left) at the low beta section L06 of BESSY II and view on the beamline (right).

This extensive range of new capabilities was enabled by a series of key improvements. We are using now a plane grating monochromator (PGM) with a highly efficient blaze grating manufactured by the HZB Department of Precision Gratings. In addition, we have chosen grazing incidence angles of $1^{\circ}$ for the pre-mirror M1 as well as for the mirror M3c. The mirrors are rhodium coated for high reflectivity 
over the whole photon energy range from $270 \mathrm{eV}$ up to $2.5 \mathrm{keV}$. The optical layout was chosen in such a way that it delivers a round shaped illumination of the condenser. In addition to this new beamline design, we will present results of the metrology measurements [6,7] of the new optical elements installed in the beamline as well as the first at wavelength measurements.

At wavelength measurements shown in Fig. 2 show the high grating efficiency within the photon energy range $(170 \mathrm{eV}-1.8 \mathrm{keV})$ provided by the U41. Figure 3 shows the measured flux curves for the first, third, fifth and seventh undulator harmonic.

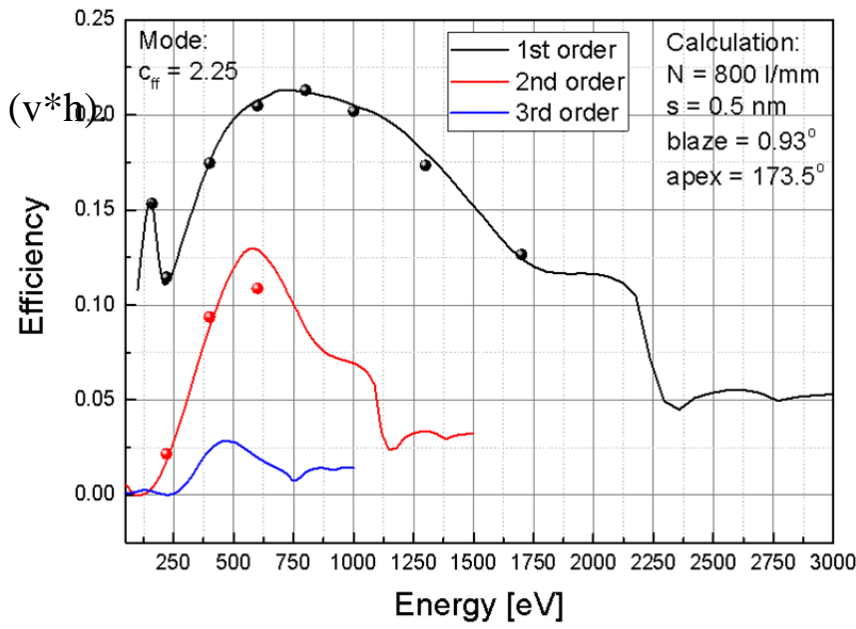

Figure. 2. Efficiency measurement of the $800 \mathrm{l} / \mathrm{mm}$ plane grating having a blaze angle of $0.93^{\circ}$ were performed with the reflectometer of the BESSY II (HZB) Optics beamline (PM1) [8]. The beam size during the measurement was $0.36 * 0.2 \mathrm{~mm}^{2}(\mathrm{v} * \mathrm{~h})$ and the detectors aperture were $0.14 * 4 \mathrm{~mm}^{2}(\mathrm{v} * \mathrm{~h})$.

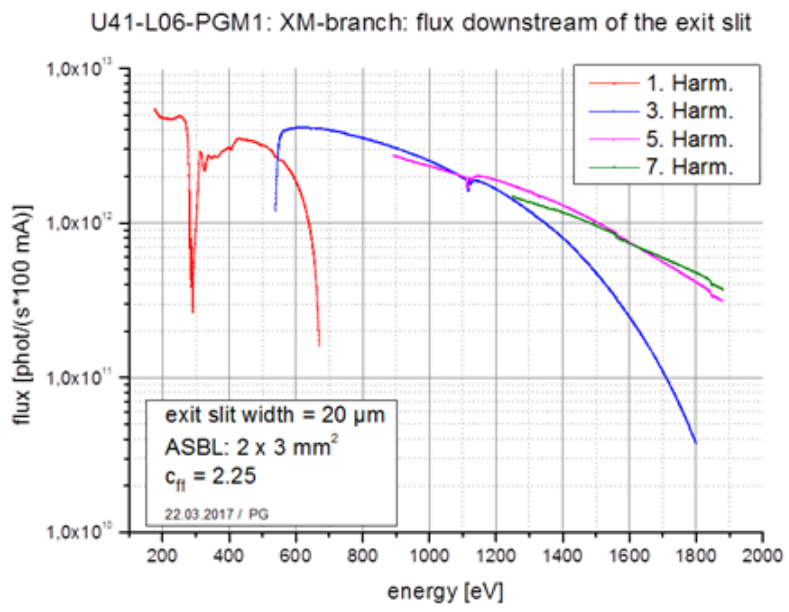

Figure. 3. Flux curves measured directly downstream of the exit slit with typical parameter settings of the front end aperture (ASBL), monochromator $\left(\mathrm{c}_{\mathrm{ff}}\right.$-value) and exit slit width.

References:

[1] G. Schneider et al, J. Struct. Biol. 177 (2012), 212-223

[2] C. Hagen et al, Cell 163 (2015), 1692-1701

[3] P. Guttmann, C. Bittencourt, Beilstein J. Nanotechnol. 6 (2015), 595-604

[4] K. Henzler et al, Scientific Reports 5, 17729 (2015)

[5] D. Carta et al, Scientific Reports 6, 21525 (2016)

[6] F. Siewert et al, Nuclear Instruments \& Methods in Physics Research A 635 (2011), p. S52 - S57

[7] A. Sokolov et al, Rev. Scientific Instruments 87 (2016), p. 052005/1-7

[8] F. Schäfers et al, J. Synchrotron Rad. 23 (2015), 67-77 